\title{
CONSTRUCTING BASES FOR RADICALS AND NILRADICALS OF LIE ALGEBRAS
}

\author{
STEPHEN MERRIN
}

(Communicated by Andreas R. Blass)

\begin{abstract}
The radical and nilradical of a finite-dimensional Lie algebra $L$ are well defined unique subspaces of $L$. Nevertheless, we show the impossibility of ever finding a general algorithm that will construct finite bases for radicals (or nilradicals) of arbitrary finite-dimensional Lie algebras. Our approach involves an investigation of the relationship between radicals of associative algebras and radicals of Lie algebras. Building on a result of Richman in the constructive theory of associative algebras, we prove that bases for radicals and nilradicals of finite-dimensional Lie algebras over a discrete field $F$ can always be constructed if and only if $F$ satisfies Seidenberg's condition P. A special case is that if we restrict ourselves to fields of characteristic zero, we can indeed always construct bases for radicals. Our proofs are entirely constructive (i.e., do not use the general law of excluded middle).
\end{abstract}

\section{INTRODUCTION}

The radical and nilradical of a finite-dimensional Lie algebra $L$ are well defined subspaces of $L$. Although it is trivially true in classical mathematics that these subspaces are finite dimensional (every subspace of $L$ is finite dimensional), the question of whether radicals are finite dimensional acquires substance if we demand that our proofs be entirely constructive. In constructive mathematics we do not use the general principle of excluded middle. Whereas in classical mathematics the logical schema $A \vee \neg A$ (read " $A$ or not $A$ ") is accepted regardless of what the formula $A$ is (e.g., classically speaking, either Fermat's Last Theorem is true or it is not), in constructive mathematics a proof of $A \vee \neg A$ must establish one of $A$ and $\neg A$, and we know which one. The schema $A \vee \neg A$ is therefore rejected in the constructive setting. To prove, using classical logic, a theorem of the form $\exists x B(x)$, it is sufficient to demonstrate that $\forall x \neg B(x)$ is impossible, but in constructive mathematics a proof of $\exists x B(x)$ must indicate how to construct an $x$ such that $B(x)$. The construction of $x$ is usually accomplished by sketching an algorithm for finding $x$. A constructive proof that a vector space is finite dimensional must indicate how to construct a finite basis of the vector space in question.

In [1] Beck, Kolman, and Stewart write:

Received by the editors June 20, 1991 and, in revised form, March 9, 1992.

1991 Mathematics Subject Classification. Primary 17B05, 03F65.

Key words and phrases. Lie algebra, constructive algebra, radical, nilradical. 
The aim of this paper is to obtain algorithms for locating in a finite-dimensional Lie algebra $L$ items of structural interest. This structural analysis will include calculating the various radicals of $L \ldots$ as well as determining whether $L$ is nilpotent, soluble, or semisimple.

It is surprising how much of the traditional mathematical theory of Lie algebras is purely existential. For example, whilst it is obvious that a maximal soluble ideal exists in $L$ and is unique, it is not immediately clear how it may be found algorithmically.

In showing how to compute a basis for the "maximal soluble ideal" of $L$ (below, we denote this ideal as $\operatorname{rad} L$ ) Beck et al. use classical logic and call upon classical results in the theory of Lie algebras. Also, importantly, they limit themselves to fields of characteristic zero, saying nothing about the finite characteristic case. In our paper we offer what we believe to be significant improvements over their results. Our (perhaps surprising) conclusion is that it is impossible to prove constructively the general theorem that asserts that radicals (or nilradicals) of finite-dimensional Lie algebras are themselves finite dimensional. In fact, we characterize those discrete fields (in constructive jargon a set $X$ is said to be discrete if the equality relation on $X$ is decidable: for all $x, y \in X$, we can tell whether or not $x=y$ ) over which we can always construct bases for the radicals. Building on a result of Richman [6, Chapter IX, Theorem 4.4] in the constructive theory of associative algebras, we show that bases for radicals and nilradicals of finite-dimensional Lie algebras over a discrete field $F$ can always be constructed if and only if $F$ satisfies Seidenberg's condition P. A special case of this is that over discrete fields of characteristic zero (e.g., the rational numbers), we can indeed always construct bases for the radicals.

The beauty of the constructive approach to Lie algebras (and to mathematics in general) is that even though our proofs are in fact ordinary mathematical proofs, they can be turned into computer programs, if so desired. Unlike Beck et al. [1], who sometimes use APL notation to express their algorithms, we constructive mathematicians are not burdened with the details of a particular programming language. The style of constructive mathematics that we follow is that of Bishop [2] and Mines, Richman, and Ruitenburg [6].

Any development of the abstract theory of Lie algebras needs to draw upon results from linear algebra. A constructive treatment of Lie algebras is made difficult by the fact that many of the well-known classical theorems of linear algebra, such as "every subspace of a finite-dimensional vector space has a finite basis", are simply unprovable constructively. Therefore, in our constructive approach it is unavoidable that we expend some effort examining the foundations of constructive mathematics. Much of the general background material that we will need can be found in Mines et al. [6]. A detailed introductory treatment of some topics in the constructive theory of Lie algebras is [5]. The authors of [6] do not treat Lie algebras at all. In the appendix we gather some of the background material that we will need.

The results in this paper are part of the author's Ph.D. dissertation, written at New Mexico State University under the guidance of Fred Richman. 


\section{Condition $\mathbf{P}$}

A field $F$ is discrete if for all $x, y \in F$ we can decide whether or not $x=y$. We say that a discrete field $F$ satisfies condition $P$ if for all primes $p$, if $F$ has characteristic $p$, then for all matrices $A \in F^{m \times n} \quad(m \geq 1$ and $n \geq 1)$, either there exists some nonzero $n \times 1$ matrix $x$ with entries in $F^{p}=\left\{y^{p}: y \in F\right\}$ such that $A x=0$, or for all $n \times 1$ matrices $x$ with entries in $F^{p}, A x=0$ implies $x=0$.

In classical algebra every field trivially satisfies condition $\mathrm{P}$, but in the constructive setting the notion of condition $P$ gains substance, as we shall see later. In regard to the use of the universal quantifier on the variable $p$ in the definition of condition $\mathrm{P}$, we comment that in the context of constructive mathematics, it is not always possible to compute the characteristic of a discrete field. The reader is referred to [6, p. 46] for an example. To use the notion of condition $P$, one does not need to be able to compute the characteristic of a field. Below we will give an example of a field $F$ for which it is impossible to show, using constructive reasoning, that $F$ satisfies condition $\mathrm{P}$.

Condition P, also called Seidenberg's condition P, is due to Seidenberg [7]. The reader is also referred to [6, Chapter VII, $\S 3]$ for more details on condition P.

Theorem 4.4 of [6, Chapter IX], due to Richman, says that a discrete field $F$ satisfies condition $\mathrm{P}$ if and only if every finite-dimensional associative $F$ algebra with identity has a finite-dimensional radical. An examination of the relationship between radicals of associative algebras and radicals of Lie algebras will enable us to use this theorem to answer some questions concerning the finite dimensionality of the radical and nilradical of a finite-dimensional Lie algebra.

We conclude our introduction to condition $\mathrm{P}$ by showing that the statement "every discrete field satisfies condition P" is not provable constructively. The field $F$ defined below is our own version of a related example in Seidenberg [7].

Let $S$ be an arbitrary mathematical statement. Let $\mathbb{Z}_{2}$ be the integers modulo 2, and let $Y$ be an indeterminate. Let $F_{1}$ be $\mathbb{Z}_{2}\left(Y^{2}\right)$, the field of quotients of $\mathbb{Z}_{2}\left[Y^{2}\right]$; let $F_{2}$ be $\mathbb{Z}_{2}(Y)$, the field of quotients of $\mathbb{Z}_{2}[Y]$. Define $F=\left\{z \in F_{2}: S \vee z \in F_{1}\right\}$. Then $F_{1} \subseteq F \subseteq F_{2}$ are discrete fields of characteristic 2 such that if $S$ then $F=F_{2}$ and if $\neg S$ then $F=F_{1}$. Note that $Y^{2} \in F$ and $Y \notin F_{1}$. Next, we verify that

$$
\exists a, b \in F\left(a, b \text { not both zero and } a^{2}+b^{2} Y^{2}=0\right) \leftrightarrow S .
$$

If not both $a$ and $b$ are zero, then $Y^{2}=-a^{2} / b^{2}=(a / b)^{2}$. Considering the equation $Y^{2}-(a / b)^{2}=0$ in $F_{2}$, we have $Y^{2}-(a / b)^{2}=(Y-a / b)^{2}=0$ and get $Y=a / b \in F$. But $Y \notin F_{1}$, so by the definition of $F$ we conclude $S$. On the other hand, if $S$ holds then $Y \in F$, and we take $a=Y$ and $b=1$.

Assuming $F$ satisfies condition $\mathrm{P}$, we let $A \in F^{1 \times 2}$ be the matrix $\left[\begin{array}{ll}1 & Y^{2}\end{array}\right]$. Then (according to the definition of condition $\mathrm{P}$ ) either we can find $a, b \in F$, not both zero, such that

$$
A\left[\begin{array}{l}
a^{2} \\
b^{2}
\end{array}\right]=0
$$

or we cannot find such $a, b$. Then, by the comments above, either $S$ holds or $\neg S$ holds. 
Thus, a constructive proof that every discrete field satisfies condition $\mathrm{P}$ leads to a constructive proof of $S \vee \neg S$. Since constructive mathematics rejects the schema $S \vee \neg S$, the conclusion is that "every discrete field satisfies condition $\mathrm{P}$ " has no constructive proof.

\section{RADicals of LiE Algebras AND ASSOCIATIVE ALGEBRAS}

Throughout this section, $F$ is a discrete field. A finite-dimensional Lie algebra $L$ over $F$ is a finite-dimensional vector space over $F$ together with an $F$-bilinear product that satisfies the "Lie conditions"

$$
[x, x]=0 \text { and }[[x, y], z]+[[z, x], y]+[[y, z], x]=0 .
$$

We are using the traditional notation $[x, y]$ to denote the product of elements $x, y$ in $L$. It is convenient to introduce the following notation. When we talk about, for example, "the product $[w, x, y, z]$, associated to the right" we will mean $[w,[x,[y, z]]]$. There is another way to associate elements that is important. We will write $\llbracket w, x, y, z \rrbracket$ to denote the product $[[w, x],[y, z]]$, and we will write $\llbracket a, b, c, d, w, x, y, z \rrbracket$ to denote $[[[a, b],[c, d]],[[w, x]$, $[y, z]]]$. In general, if $x_{1}, \ldots, x_{m}$ are elements in $L$ and $m$ is a power of 2 , $\llbracket x_{1}, \ldots, x_{m} \rrbracket$ is defined recursively by

$$
\begin{aligned}
\llbracket x_{1} \rrbracket & =x_{1}, \\
\llbracket x_{1}, \ldots, x_{s} \rrbracket & =\left[\llbracket x_{1}, \ldots, x_{r} \rrbracket, \llbracket x_{r+1}, \ldots, x_{s} \rrbracket\right],
\end{aligned}
$$

where $s=2^{k+1}$ and $r=2^{k}$, for some $k \geq 0$.

If $I$ is a subspace of $L$ and $k \geq 1$, define $I^{k}$ to be the set of all sums of products $\left[x_{1}, x_{2}, \ldots, x_{k}\right]$, associated to the right, where the $x_{i}$ 's are in $I$. If $k \geq 0$, define $I^{(k)}$ to be the set of all sums of products $\llbracket x_{1}, x_{2}, \ldots, x_{r} \rrbracket$, where $r=2^{k}$ and the $x_{i}$ 's are in $I$. We say $I$ is nilpotent if $I^{k}=0$ for some $k \geq 1$; we say $I$ is solvable if $I^{(k)}=0$ for some $k \geq 0$.

If $L$ is a Lie algebra and $\operatorname{End}_{F}(L)$ is the collection of all linear transformations on the vector space $L$, we define ad: $L \rightarrow \operatorname{End}_{F}(L)$ by letting ad $x$ be the map $(\operatorname{ad} x)(y)=[x, y]$, for all $x$ and $y$ in $L$.

If $L$ is an $n$-dimensional Lie algebra, the radical of $L$, written $\operatorname{rad} L$, is defined to be the union over all solvable ideals of $L$. The nilradical of $L$, written nilrad $L$, is defined to be the union over all nilpotent ideals of $L$. Since the sum of solvable ideals is a solvable ideal and the sum of nilpotent ideals is a nilpotent ideal, both $\operatorname{rad} L$ and nilrad $L$ are ideals of $L$. We show in the appendix that

$$
(\operatorname{rad} L)^{(n)}=(\operatorname{nilrad} L)^{n+1}=0 .
$$

Using the Lie conditions, it can be shown that every nilpotent ideal is also a solvable ideal, and it follows that nilrad $L \subseteq \operatorname{rad} L$. Furthermore, $\operatorname{nilrad} L=0$ implies $\operatorname{rad} L=0$. To see this, assume every nilpotent ideal of $L$ is 0 . Then, using the fact that $I^{(k+1)}=\left(I^{(k)}\right)^{2}$, it is easy to prove by induction on $k \geq 0$ that, for all ideals $I$ of $L$, if $I^{(k)}=0$ then $I=0$. Therefore, $\operatorname{rad} L=0$.

If $A$ is an associative $F$-algebra and $I$ is a subspace of $A$, define $I^{k}$, where $k \geq 1$, to be the set of all sums of products $a_{1} a_{2} \cdots a_{k}$, where the $a_{i}$ 's are in $I$. We say that $I$ is nilpotent if $I^{k}=0$ for some $k \geq 1$. A bit more generally, a subset $S$ of $A$ is said to be nilpotent if there is some $k \geq 1$ such that the 
product $s_{1} s_{2} \cdots s_{k}$ is zero, for all $s_{1}, \ldots, s_{k}$ in $S$. If $A$ is finite dimensional, we define the radical of $A$, written $\operatorname{rad} A$, to be the union over all nilpotent ideals of $A$. Then $\operatorname{rad} A$ is an ideal of $A$ and $(\operatorname{rad} A)^{n+1}=0$, where $n$ is the dimension of $A$.

Let $L$ be a finite-dimensional Lie algebra over $F$. Jacobson [3, $\S 3$, Chapter II], gives the following formula expressing the nilradical of $L$ in terms of the radical of an associative algebra: $\operatorname{nilrad} L=\operatorname{ad}^{-1}(\operatorname{rad} A)$, where $A$ is the subalgebra of the associative algebra $\operatorname{End}_{F}(L)$ (multiplication is composition) generated by $\left\{f \in \operatorname{End}_{F}(L): f=1\right.$ or $f=\operatorname{ad} x$ for some $\left.x \in L\right\}$. Observe that $A$ is also generated by the finite set $\left\{1, \operatorname{ad} e_{1}, \ldots\right.$, ad $\left.e_{n}\right\}$, where $e_{1}, \ldots, e_{n}$ is a basis of the vector space $L$. See the appendix for the justification that $A$ is therefore finite dimensional. Unfortunately, Jacobson's proof of this formula for nilrad $L$ has many nonconstructive aspects. A completely constructive treatment of Jacobson's formula and related theorems was undertaken in $[4,5]$, resulting in the following constructive theorems:

Theorem 1. Let $V$ be a finite-dimensional vector space over $F$. Suppose $S$ is a subset of $\operatorname{End}(V)$ such that

$$
\forall f, g \in S \exists \lambda \in F(f g+\lambda g f \in S) .
$$

Then for every finite subset $W$ of $S$, either $W$ is nilpotent as a subset of the associative algebra $\operatorname{End}(V)$ (multiplication is composition) or there exists some transformation in $S$ that is not nilpotent.

Theorem 2. Let $V$ be a finite-dimensional vector space over $F$, and let $B \subseteq$ $W \subseteq \operatorname{End}(V)$ be subsets such that

(1) each member of $B$ is a nilpotent transformation, and

(2) $\forall b \in B \forall w \in W \exists \lambda \in F(b w+\lambda w b \in B)$.

If $W^{+}$is the subalgebra of the associative algebra $\operatorname{End}(V)$ generated by $W$, then $B \subseteq \operatorname{rad} W^{+}$. (Note: $W^{+}$is the span of all products $w_{1} w_{2} \cdots w_{k}$, where $k \geq 1$ and the $w_{i}$ 's are in $W$.)

Theorem 2 can be proved using Theorem 1. A constructive verification of Jacobson's formula for nilrad $L$ is an easy consequence of Theorem 2, where $V$ is the vector space $L, B$ is $\operatorname{ad}(\operatorname{nilrad} L)$, and $W$ is $(\operatorname{ad} L) \cup\{1\}$.

\section{THE MAIN THEOREM}

Our principal goal is to answer the following question: can we construct, in general, bases for the radical and nilradical of a finite-dimensional Lie algebra over a discrete field? In order to answer "yes" we must spell out the construction of these bases. A "no" answer, however, is trickier, since this amounts to an independence result in intuitionistic logic. The next theorem will settle the matter.

Theorem. Let $\mathfrak{L}$ be the class of all finite-dimensional Lie algebras over the discrete field $F$, and let $\mathfrak{A}$ be the class of all finite-dimensional associative $F$ algebras with identity. Then the following statements are equivalent:

(1) $F$ satisfies condition $P$.

(2) For all $A$ in $\mathfrak{A}, \operatorname{rad} A$ is finite dimensional.

(3) For all commutative $A$ in $\mathfrak{A}, \operatorname{rad} A$ is finite dimensional. 
(4) For all $L$ in $\mathfrak{L}$, nilrad $L$ is finite dimensional.

(5) For all $L$ in $\mathfrak{L}, \operatorname{rad} L$ is finite dimensional.

(6) For all $L$ in $\mathfrak{L}, \operatorname{rad} L=0$ or $\operatorname{rad} L \neq 0$.

(Note: $\operatorname{rad} L \neq 0$ means we can construct a nonzero $x$ in $L$ such that $x \in$ $\operatorname{rad} L$.)

Proof. Items (1)-(3) are proven equivalent in [6, p. 244]. Of the remaining implications to show, perhaps the most interesting is $(5) \rightarrow(3)$. Let $A$ be a commutative algebra in $\mathfrak{A}$. Consider the Lie algebra $H \otimes A$, where $H$ is the 3-dimensional "cross product" Lie algebra over $F$ with basis $r, s, t$ such that $[r, s]=t,[s, t]=r$, and $[t, r]=s$. The Lie algebra multiplication in the tensor product $H \otimes A$ is determined by $[x \otimes a, y \otimes b]=[x, y] \otimes a b$, for all $x, y \in H$ and $a, b \in A$. Let $D=\{u \in H \otimes A: \exists a, b, c \in \operatorname{rad} A(u=$ $r \otimes a+s \otimes b+t \otimes c)\}$. Note that $D$ is the image of the natural embedding of $H \otimes \operatorname{rad} A$ in $H \otimes A$ (see [6, Chapter III] for a constructive presentation of tensor products). Let $m=\operatorname{dim} A$. Since $(\operatorname{rad} A)^{m+1}=0$, it follows that $D^{m+1}=0$, and we conclude that $D$ is a nilpotent ideal of the Lie algebra $H \otimes A$ and, hence, is contained in $\operatorname{nilrad}(H \otimes A)$. Our next step is to show that $\operatorname{rad}(H \otimes A) \subseteq D$, from which it will follow that $\operatorname{rad}(H \otimes A)=D=\operatorname{nilrad}(H \otimes A)$. Let $I$ be any ideal of $H \otimes A$, and define $J$ to be the set

$$
\{x \in A: \exists a, b, c \in A(r \otimes a+s \otimes b+t \otimes c \in I \text { and } x \in\{a, b, c\})\} .
$$

We first observe that if $u=r \otimes a+s \otimes b+t \otimes c \in I$, then for all pairs $(x, y)$. in $\{r, s, t\} \times\{a, b, c\}, x \otimes y$ lies in $I$. To see this, we compute the following products, always associating to the right:

$$
\begin{aligned}
{[r \otimes 1, t \otimes 1, u] } & =t \otimes a, \\
{[s \otimes 1, r \otimes 1, t \otimes 1, u] } & =r \otimes a, \\
{[t \otimes 1, s \otimes 1, r \otimes 1, t \otimes 1, u] } & =s \otimes a, \\
{[s \otimes 1, r \otimes 1, u] } & =r \otimes b, \\
{[t \otimes 1, s \otimes 1, r \otimes 1, u] } & =s \otimes b, \\
{[r \otimes 1, t \otimes 1, s \otimes 1, r \otimes 1, u] } & =t \otimes b, \\
{[t \otimes 1, s \otimes 1, u] } & =s \otimes c, \\
{[r \otimes 1, t \otimes 1, s \otimes 1, u] } & =t \otimes c, \\
{[s \otimes 1, r \otimes 1, t \otimes 1, s \otimes 1, u] } & =r \otimes c .
\end{aligned}
$$

Since $I$ is an ideal, all these products lie in $I$.

From the preceding observation it follows that $J=\{a \in A: r \otimes a \in I\}$. It is now easy to show that $J$ is an ideal of the associative algebra $A$. The final step in establishing $\operatorname{rad}(H \otimes A) \subseteq D$ is to show that if $I$ is a solvable ideal then $J$ is a nilpotent ideal. Assume $I^{(k)}=0$ for some $k \geq 0$. We will show that $J^{m}=0$, where $m=2^{k}$. Let $a_{1}, a_{2}, \ldots, a_{m} \in J$. Let $T$ be the infinite binary tree with nodes labeled by $r, s$, or $t$, such that $r$ is at the root, and, for each node $n$ of $T$, the label of $n$ is the product (in the Lie algebra $H$ ) of the labels of the left and right children of $n$. Part of $T$ is shown in the following 
diagram:

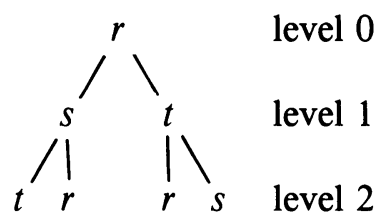

Let $x_{1}, x_{2}, \ldots, x_{m}$ be the labels (from left to right) of the nodes at level $k$ of the tree. By the observations in the previous paragraph concerning the ideal $I$, the elements $x_{1} \otimes a_{1}, x_{2} \otimes a_{2}, \ldots, x_{m} \otimes a_{m}$ are all in $I$. Then

$$
r \otimes a_{1} a_{2} \cdots a_{m}=\llbracket x_{1} \otimes a_{1}, x_{2} \otimes a_{2}, \ldots, x_{m} \otimes a_{m} \rrbracket \in I^{(k)}=0 .
$$

The product $\llbracket \cdots \rrbracket$ is carried out by working our way up the tree $T$, starting at level $k$ and ending at the root. Since $r \otimes a_{1} a_{2} \cdots a_{m}=0$ implies $a_{1} a_{2} \cdots a_{m}=0$, we are done.

In summary, if $I$ is any solvable ideal of $H \otimes A$ then the corresponding ideal $J$ of $A$ is nilpotent and, hence, is contained in $\operatorname{rad} A$. Therefore, by the definition of $D$ and $J$, we obtain $I \subseteq D$, which shows that $\operatorname{rad}(H \otimes A) \subseteq D$.

We conclude the proof of $(5) \rightarrow(3)$ by noting that $D=\operatorname{rad}(H \otimes A)$ is finite dimensional by assumption (5). But $H \otimes \operatorname{rad} A \cong D$ and so $H \otimes \operatorname{rad} A$ is finite dimensional. The finite dimensionality of both $H$ and $H \otimes \operatorname{rad} A \operatorname{implies} \operatorname{rad} A$ is finite dimensional. This last statement is a consequence of the following easyto-show result about tensor products. If $V$ and $W$ are vector spaces over $F$ such that both $V \otimes W$ and $V$ are finite dimensional, with $\operatorname{dim} V \geq 1$, then $W$ is finite dimensional.

We prove $(6) \rightarrow(5)$ by induction on the dimension of $L$. If $L$ is in $\mathfrak{L}$, then $\operatorname{rad} L=0$ or $\operatorname{rad} L \neq 0$ by (6). If $\operatorname{rad} L=0$ then $\operatorname{dim} \operatorname{rad} L=0 ;$ if $\operatorname{rad} L \neq 0$, we can find some nonzero $x$ in $\operatorname{rad} L$. Let $I$ be the (solvable) ideal of $L$ generated by $x$. Then $I$ and $L / I$ are finite dimensional and $\operatorname{dim} L / I<\operatorname{dim} L$. By induction, $\operatorname{rad}(L / I)$ is finite dimensional. Write $\operatorname{rad}(L / I)=J / I$ for some ideal $J$ of $L$ containing $I$. Since $J / I$ and $I$ are both finite dimensional and solvable, then $J$ is finite dimensional and solvable. It is easy to check that in fact $J=\operatorname{rad} L$, which proves that $\operatorname{rad} L$ is finite dimensional.

The implication $(4) \rightarrow(6)$ is easy, once we recall from $\S 3$ that $\operatorname{nilrad} L \subseteq$ $\operatorname{rad} L$ and that $\operatorname{nilrad} L=0$ forces $\operatorname{rad} L=0$.

If $L \in \mathfrak{L}$ and we let $A$ be the subalgebra of the associative algebra $\operatorname{End}_{F}(L)$ generated by $\left\{1\right.$, ad $e_{1}, \ldots$, ad $\left.e_{n}\right\}$, where $e_{1}, \ldots, e_{n}$ is a basis of the vector space $L$, then the proof of $(2) \rightarrow(4)$ follows from the formula nilrad $L=$ $\operatorname{ad}^{-1}(\operatorname{rad} A)$ given in $\S 3$.

\section{Conclusion}

According to our discussion in $\S 2$, it is not possible to prove constructively that an arbitrary discrete field satisfies condition $P$. Therefore, by the previous theorem, it is impossible to devise constructive proofs of (2)-(6) of the theorem, for an arbitrary discrete field $F$. In particular, it is fruitless to look for a general algorithm that will construct bases of the radical and nilradical of a finite-dimensional Lie algebra. It is also impossible to devise a general procedure that will decide whether or not a finite-dimensional Lie algebra is semisimple (i.e., whether or not its radical is 0 ). On the other hand, since a discrete field 
of characteristic zero (vacuously) satisfies condition $\mathrm{P}$, we can indeed find such algorithms if we restrict ourselves to algebras over discrete fields of characteristic zero.

Although we cannot always find bases for the radical and nilradical, these subspaces are nevertheless detachable. That is, given an arbitrary element $x$ in a finite-dimensional Lie algebra $L$, we can always determine whether or not $x$ is a member of $\operatorname{rad} L$ and whether or not $x$ is a member of nilrad $L$. The procedure to do this is as follows. Given $x \in L$, we let $C$ be the (finitedimensional) ideal of $L$ generated by $x$. We can then test whether or not $C$ is nilpotent and whether or not $C$ is solvable (see the appendix). Finally, $x$ is in $\operatorname{rad} L$ if and only if $C$ is solvable, and $x$ is in nilrad $L$ if and only if $C$ is nilpotent.

\section{APPENDIX}

Linear algebra. The following paragraph lists some elementary results in constructive linear algebra that we made use of in the preceding sections.

If $V$ and $W$ are finite-dimensional vector spaces over a discrete field $F$ and $T: V \rightarrow W$ is a linear transformation, then both $\operatorname{ker} T$ and $\operatorname{Im} T$ are finite dimensional. As shown in [6], finite bases for $\operatorname{ker} T$ and $\operatorname{Im} T$ can be obtained by keeping track of the row and column operations needed to put the matrix of $T$ into a certain standard form. The preceding result has many applications, some of which we indicate next. If $T, V$, and $W$ are as before and $U$ is a finite-dimensional subspace of $W$, then the quotient $W / U$ is finite dimensional and $T^{-1} U$ is a finite-dimensional subspace of $V$. A subspace of a finite-dimensional space is finite dimensional if and only if the subspace is finitely generated. The intersection of two finite-dimensional subspaces of a finite-dimensional vector space is again finite dimensional.

Nonassociative algebras. We now give constructive proofs of some basic facts concerning Lie and associative algebras used previously but whose proofs have been postponed until now. A nonassociative algebra over a discrete field $F$ is a vector space over $F$ together with an $F$-bilinear product. The description "nonassociative" means "not necessarily associative". Lie algebras and associative algebras are special kinds of nonassociative algebras. The multiplication in a Lie algebra satisfies the "Lie conditions", and multiplication in an associative algebra satisfies the associative law.

We will usually write the product of two elements $x, y$ in a nonassociative algebra $A$ as $x y$, although if $A$ is a Lie algebra it is common to write the product as $[x, y]$. If $A$ is a finite-dimensional nonassociative algebra and $B$ and $W$ are subsets of $A$, define $B^{*} W$ to be the subspace of $A$ spanned by all products $b_{1} b_{2} \cdots b_{k} w$, associated to the right, where the $b_{i}$ 's are in $B, w$ is in $W$, and $k \geq 0$ (e.g., the product $b_{1} b_{2} \cdots b_{k} w$, associated to the right, is $w$ when $k=0, b_{1} w$ when $k=1, b_{1}\left(b_{2} w\right)$ when $k=2$, and $b_{1}\left(b_{2}\left(b_{3} w\right)\right)$ when $k=3$ ). We will write $W^{*} W$ as $W^{+}$. Note that $W^{+}$is the span of all products, associated to the right, of one or more elements of $W$. Observe that $A^{*} W$ is the left ideal of $A$ generated by the set $W$. If $B$ is a subset of $A$ that spans $A$, then $A^{*} W=B^{*} W$. If $A$ is an associative algebra, then it is clear that $W^{+}$is closed under products. In the case that $A$ is a Lie algebra, 
one can use the Lie conditions to show that $W^{+}$is closed under products. In either case $W^{+}$is the subalgebra of $A$ generated by $W$.

Theorem A.1. If $B$ and $W$ are finite subsets of the finite-dimensional nonassociative $F$-algebra $A$, then $B^{*} W$ is a finite-dimensional subspace of $A$.

Proof. For each $k \geq 0$, define $B^{k} W$ to be the subspace of $A$ spanned by the (finite) collection of all products $b_{1} b_{2} \cdots b_{j} w$, associated to the right, where the $b_{i}$ 's are in $B, w$ is in $W$, and $0 \leq j \leq k$. Then the family $\left\{B^{k} W\right\}_{k \geq 0}$ is an ascending chain of finite-dimensional subspaces of $A$ whose union is $B^{*} W$. Let $n=\operatorname{dim} A$. It cannot happen that $\operatorname{dim} B^{i} W<\operatorname{dim} B^{i+1} W$ for all $0 \leq i \leq n$, so we can find some $0 \leq k \leq n$ such that $B^{k} W=B^{k+1} W$. One can check that $B^{k} W=B^{k+r} W$ for all $r \geq 1$. Thus, $B^{*} W=B^{k} W$, proving that $B^{*} W$ is finite dimensional.

As a corollary, if $W$ is a finite subset of the finite-dimensional nonassociative algebra $A$, then both $W^{+}$and the left ideal generated by $W$ are finitedimensional subspaces of $A$.

If $S$ is a subset of the nonassociative algebra $A$, we say that $S$ is nilpotent if and only if there exists some $m \geq 1$ such that the product $s_{1} s_{2} \cdots s_{m}$, associated to the right, is zero, for all $s_{1}, \ldots, s_{m}$ in $S$. The following theorem says that if $A$ is finite dimensional, then there is a uniform bound on the "nilpotency" $m$.

Theorem A.2. Let $A$ be an $n$-dimensional nonassociative $F$-algebra and $S$ a subset of $A$. Then the following are equivalent:

(1) $S$ is nilpotent.

(2) For all $s_{1}, s_{2}, \ldots, s_{n+1} \in S$, the product $s_{1} s_{2} \cdots s_{n+1}$, associated to the right, is zero.

(3) Every finite subset of $S$ is nilpotent.

Proof. The implications $(2) \rightarrow(1)$ and $(1) \rightarrow(3)$ are immediate. To show $(3) \rightarrow(2)$, suppose $s_{1}, s_{2}, \ldots, s_{n+1} \in S$ and let $T=\left\{s_{1}, s_{2}, \ldots, s_{n+1}\right\}$. According to assumption (3), there is some $m \geq 1$ such that $t_{1} t_{2} \cdots t_{m}$, associated to the right, is zero, for all $t_{1}, t_{2}, \ldots, t_{m}$ in $T$. For each $k \geq 1$, define $T_{k}$ to be the subspace of $A$ spanned by the set of all products $t_{1} t_{2} \cdots t_{j}$, associated to the right, where $j \geq k$ and the $t_{i}$ 's are in $T$. The family $\left\{T_{k}\right\}_{k \geq 1}$ is a descending chain of subspaces of $A$. Note that $T_{k}=0$ for all $k \geq m$. We now prove by induction on $k \geq 1$ that $T_{k}$ is a finitely generated subspace of $A$. Observing that $T_{1}$ is the same as $T^{+}$, the case $k=1$ follows from Theorem A.1. By induction, assume there is a finite set $X \subseteq T_{k}$ that spans $T_{k}$. Then $T_{k+1}$ is spanned by the finite set $\{a b: a \in T$ and $b \in X\}$.

We have now established that $\left\{T_{k}\right\}_{k \geq 1}$ is a descending chain of finitedimensional subspaces of $A$. Since $\operatorname{dim} A=n$, it cannot happen that $\operatorname{dim} T_{k+1}$ $<\operatorname{dim} T_{k}$ for all $1 \leq k \leq n+1$, so we can find some $1 \leq k_{0} \leq n+1$ such that $T_{k_{0}+1}=T_{k_{0}}$. It follows by induction on $r$ that $T_{k_{0}+r}=T_{k_{0}}$ for all $r \geq 1$. Recalling that $T_{k}=0$ for all $k \geq m$, we find that $T_{k_{0}}=0$. Since $k_{0} \leq n+1$, we see that the product $s_{1} s_{2} \cdots s_{n+1}$, associated to the right, lies in $T_{k_{0}}=0$, as desired.

There are several important applications of Theorem A.2. If $A$ is an $n$ dimensional associative algebra, then $(\operatorname{rad} A)^{n+1}=0$. If $L$ is an $n$-dimensional 
Lie algebra, then $(\operatorname{nilrad} L)^{n+1}=0$. It also follows from Theorem A.2 that if $S$ is a finite-dimensional subspace of an $n$-dimensional nonassociative $F$-algebra, then it is decidable whether or not $S$ is nilpotent: if $S$ is spanned by the finite set $X, S$ is nilpotent if and only if $x_{1} x_{2} \cdots x_{n+1}$, associated to the right, is zero, for all $x_{1}, x_{2}, \ldots, x_{n+1}$ in $X$.

Theorem A.3. If $L$ is an m-dimensional Lie algebra, then $L$ is solvable if and only if $L^{(m)}=0$.

Proof. The sequence $\left\{L^{(k)}\right\}_{k \geq 0}$ is a descending chain of finite-dimensional subspaces of $L$. Since $\operatorname{dim} L=m$, it cannot be the case that $\operatorname{dim} L^{(i+1)}<\operatorname{dim} L^{(i)}$ for all $0 \leq i \leq m$, so there must be some $0 \leq k \leq m$ such that $L^{(k+1)}=L^{(k)}$. It is then the case that $L^{(k+r)}=L^{(k)}$ for all $r \geq 1$. Assuming $L$ is solvable, there is some $n_{0} \geq 0$ such that $L^{(n)}=0$ for all $n \geq n_{0}$. Then $L^{(m)}=L^{(k)}=0$.

We give two applications of this theorem. Let $L$ be an $n$-dimensional Lie algebra. We will use the [ , ] notation for Lie algebra multiplication, as in §3. The first corollary of the theorem is $(\operatorname{rad} L)^{(n)}=0$, which is shown by considering the (solvable, finite-dimensional) ideal $I$ generated by arbitrary elements $x_{1}, x_{2}, \ldots, x_{r}\left(r=2^{n}\right)$ in $\operatorname{rad} L$ and then noting by Theorem A.3 that $\llbracket x_{1}, x_{2}, \ldots, x_{r} \rrbracket \in I^{(n)}=0$. Second, if $X$ is a finite basis of $L$, then, by Theorem A.3, $L$ is solvable if and only if $\llbracket x_{1}, x_{2}, \ldots, x_{r} \rrbracket$ is zero, for all $x_{1}, x_{2}, \ldots, x_{r}$ in $X$, where $r=2^{n}$. Thus, we can always decide whether or not a finite-dimensional Lie algebra is solvable.

\section{REFERENCES}

1. R. Beck, B. Kolman, and I. Stewart, Computing the structure of a Lie algebra, Computers in Nonassociative Rings and Algebras (R. Beck and B. Kolman, eds.), Academic Press, New York, 1977, pp. 167-188.

2. E. Bishop, Foundations of constructive analysis, McGraw-Hill, New York, 1967.

3. N. Jacobson, Lie algebras, Dover, New York, 1979.

4. S. Merrin, A strong constructive version of Engel's theorem, submitted.

5. __, Some constructive results in the theory of Lie algebras, Ph.D. dissertation, New Mexico State Univ., 1990.

6. R. Mines, F. Richman, and W. Ruitenburg, A course in constructive algebra, Springer-Verlag, New York, 1988.

7. A. Seidenberg, Construction of the integral closure of a finite integral domain, Rend. Sem. Mat. Fis. Milano 40 (1970), 100-120.

Department of Mathematics and Physical Sciences, Embry-Riddle Aeronautical UniVersity, Prescott, ARIZONA 86301

Current address: P. O. Box 278, Springville, Utah 84663-0278 